\title{
Diversidade das pescarias realizadas com rede de emalhar em um estuário tropical do nordeste do Brasil
}

\author{
Severino Adriano de Oliveira Lima ${ }^{1 *}$ \\ Humber Agrelli Andrade ${ }^{2}$ \\ Marcondes Agostinho Gonzaga Junior ${ }^{1}$ \\ Donovan Filipe Henrique Pinto ${ }^{1}$ \\ ${ }^{1}$ Universidade Federal de Rondônia, Departamento de Engenharia de Pesca \\ Rua da Paz, 4.376, Lino Alves Teixeira, CEP 76.916-000, Presidente Médici - RO, Brasil \\ ${ }^{2}$ Universidade Federal Rural de Pernambuco, Departamento de Pesca e Aquicultura \\ Rua Dom Manoel de Medeiros, Dois Irmãos, s/n, CEP 52.171-900, Recife - PE, Brasil \\ * Autor para correspondência \\ limasao@unir.com
}

Submetido em 04/10/2020

Aceito para publicação em 14/12/2020

\section{Resumo}

O objetivo neste trabalho foi estudar a diversidade da ictiofauna capturada com a rede de emalhar mais utilizada no estado de Pernambuco. No total foram realizadas 14 coletas no Canal de Santa Cruz (CSC) entre os meses de julho de 2013 e junho de 2014. As amostras foram obtidas com rede de emalhar de malha de $30 \mathrm{~mm}$. Os peixes capturados foram retirados e separados na posição margem e centro, e na posição vertical da rede (superior, meio e inferior). Quanto à frequência de ocorrência, as espécies foram classificadas como comuns, acessórias ou ocasionais. Foram realizadas análises de diversidade alfa e beta. No total foram capturadas 19 espécies, sendo Opisthonema oglinum e Anchovia clupeoides as únicas constantes. As diversidades alfa estimadas não apresentaram diferenças significativas nos estratos temporais e espaciais. Na diversidade beta dois grupos se distinguiram, sendo que no maior ocorreu geralmente grandes abundâncias de $O$. oglinum. A rede avaliada foi eficiente para a espécie alvo O. oglinum e as capturas de outras espécies corresponderam a menos de $13 \%$ da icitiofauna já encontrada no CSC. Portanto, medidas de gestão que visem restringir o uso do petrecho para a proteção da diversidade, considerando a sazonalidade e espacialidade no canal, não são recomendadas.

Palavras-chave: Engraulidae; Opisthonema oglinum; Pequenos pelágicos; Pesca estuarina

\section{Abstract}

Diversity of fisheries performed with gillnet in a tropical estuary of northeastern Brazil. The aim of the present research was to study the diversity of the ichthyofauna captured with the most commonly used gillnet in the state of Pernambuco. In total, 14 collections were made in the Santa Cruz Canal (CSC) between July 2013 and June 2014. The samples were collected using a $30 \mathrm{~mm}$ mesh gillnet. The captured fish were removed and separated in margin and center positions, as well as in the net's vertical position (upper, middle, and lower). Concerning frequency of occurrence, the species were classified as common, accessory, or occasional. Alpha and beta diversity analyses were performed. In total, 19 species were captured, with Opisthonema oglinum 
and Anchovia clupeoides being the only constants. The estimated alpha diversities did not show significant differences in the temporal and spatial strata. In the beta diversity, two groups were distinguished, with the largest generally occurring in large abundances of $O$. oglinum. The evaluated net was efficient for the target specie $O$. oglinum, while the catches of other species corresponded to less than $13 \%$ of the icitiofauna already found in the CSC. Therefore, management measures that aim to restrict the use of fishing gear for the protection of diversity considering the seasonality and spatiality in the channel is not recommended.

Key words: Engraulidae; Estuarine fishing; Opisthonema oglinum; Small pelagics

\section{Introdução}

Os estuários são habitats delimitados pela influência da maré e ocorrem nas bacias dos rios, estabelecendo limites espaciais dinâmicos (ALCÂNTARA, 2004). A interface desse ambiente é influenciada pela interação entre processos limnológicos, oceanográficos e antropogênicos (MÉRIGOT et al., 2016). A maioria dos estuários sofre com impactos humanos de origens diversas, como a destruição do ecossistema manguezal, assoreamento e descarga de efluentes urbanos (JESUS et al., 2004). Essa pressão antrópica pode interferir diretamente nos recursos alimentares, distribuição, diversidade, reprodução, abundância, crescimento, sobrevivência e comportamento dos peixes (WHITFIELD; ELLIOTT, 2002). Os peixes representam um dos principais componentes entre as espécies estuarinas nectônicas e contribuem ecologicamente para a transferência de energia nesses ambientes (POTTER et al., 2011), pois os estuários são importantes ambientes de transição para o movimento da ictiofauna entre as bacias continentais e o oceano (RAY, 2005).

A variação sazonal da composição de espécies da ictiofauna em ambientes estuarinos está relacionada fortemente com a pluviosidade (MORENOVALCÁRCEL et al., 2013), já que esta oscila em função do influxo de água doce continental, condicionada ao aporte dos períodos de seca e chuva (HAEDRICH, 1983). A sazonalidade é responsável por mudanças nos fatores ambientais que são condicionantes para a reprodução e afetam as assembleias de peixes nos estuários (MCERLEAN et al., 1973). Além do componente sazonal, a variação da ictiofauna também depende das condições espaciais, até em um mesmo estuário, que pode ter dois ou mais ambientes morfologicamente distintos como, por exemplo, as regiões rasas e protegidas, com profundidades menores que ficam situadas nas margens, e a região de águas abertas no corpo central do estuário, com maiores profundidades no leito do rio (SANTOS et al., 2015).

Os estudos ecológicos em estuários abordando as comunidades de peixes são fundamentais para a compreensão do funcionamento de todos os seus ecossistemas (BARLETTA et al., 2003). As estimativas de diversidade são ferramentas que propiciam a melhor compreensão das comunidades em vários tipos de ambientes, inclusive estuarinos. Há diferentes classificações de índices ecológicos, umas das mais usuais são as abordagens de diversidade alfa, que se refere à estimativa local de uma comunidade, e a diversidade beta, que é uma medida da diferença (ou da semelhança) entre comunidades (WHITTAKER, 1972). As estimativas de diversidade alfa podem expressar a variabilidade das espécies, domínio e equitabilidade, e esses índices são indicadores de biodiversidade que estão frequentemente associados como referências para quantificar e qualificar a variedade de populações aquáticas (VYAS et al., 2012).

Os meios de capturas utilizados para levantamento da diversidade das comunidades aquáticas são variados e entre eles têm destaque as redes de emalhar, cuja captura ocorre primordialmente em função do deslocamento do peixe. Essas redes podem ser utilizadas com diferentes tamanhos de malhas ou em conjunto com outros petrechos para o inventário da ictiofauna (e.g. SANTOS et al., 2015; ARAÚJO et al., 2017; FERREIRA et al., 2019). As redes de emalhar são amplamente utilizadas nas pescarias estuarinas, por causa do baixo custo e da facilidade de manuseio, fatores que contribuíram para a popularidade desse tipo de petrecho (HOVGÅRD et al., 1999). No Nordeste do Brasil, esse petrecho é o mais empregado nas capturas de pequenos peixes 
pelágicos, principalmente a espécie Opisthonema oglinum (NÓBREGA et al., 2009). As pescarias dessa espécie no estado de Pernambuco (Nordeste do Brasil) são efetuadas quase em sua totalidade no complexo estuarino do Canal de Santa Cruz (CSC) com emprego de redes de emalhar com tamanho de malha de 30 $\mathrm{mm}$ (entre nós opostos). Essas redes rendem maiores capturas em toneladas, quando comparadas às demais pescarias de emalhe (ANDRADE; SILVA, 2013), e têm incidência nos indivíduos juvenis (LESSA et al., 2008; LIMA; ANDRADE, 2018a). O CSC é berçário para várias espécies de peixes (BARRETO; SANTANABARRETO, 1980), mas é uma região com intenso impacto antrópico (MONTES et al., 2002; MOURA, 2009). Portanto, o objetivo neste trabalho foi estudar a diversidade da ictiofauna capturada com a rede de emalhar mais utilizada no CSC.

\section{Material e Métodos}

\section{Área de estudo}

A Área de Proteção Ambiental (APA) de Santa Cruz, localizada no extremo norte do estado de Pernambuco (Nordeste do Brasil), foi criada no ano de 2008, englobando os municípios de Itapissuma, Ilha de Itamaracá e Goiana. A finalidade da criação dessa APA foi a de proteger a diversidade biológica, mitigar a ocupação humana já estabelecida e garantir a sustentabilidade do uso dos recursos naturais, considerando três áreas estuarinas, sendo uma dessas o Complexo Estuarino Canal de Santa Cruz (CSC) (Figura 1). O CSC é o maior e mais fértil ecossistema estuarino do litoral pernambucano e o seu canal principal é um braço de mar com $22 \mathrm{~km}$ de extensão que recebe aportes fluviais

FIGURA 1: Complexo estuarino do Canal de Santa Cruz (CSC) e os municípios englobados (Ilha de Itamaracá, Itapissuma e Goiana).

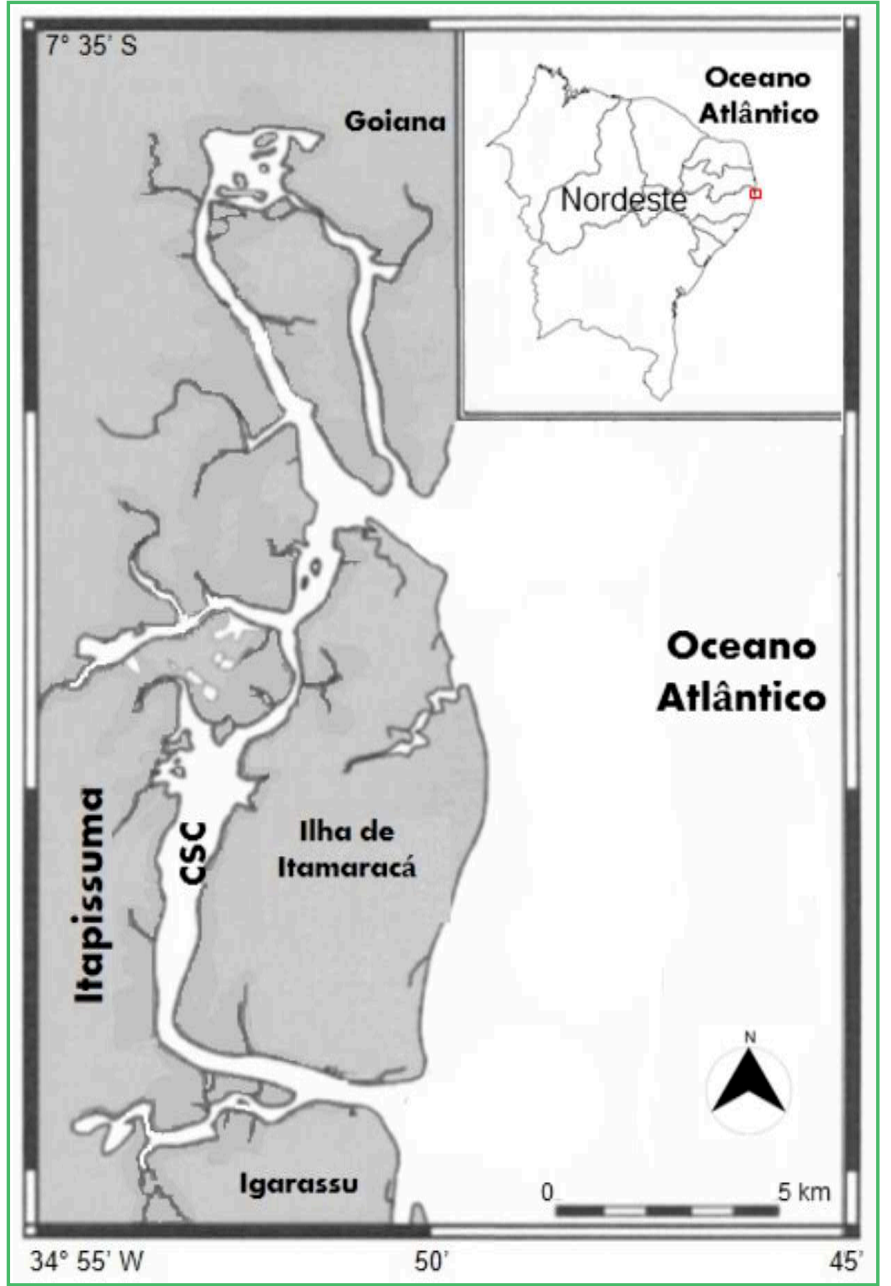


dos estuários dos rios Igarassu, Botafogo, Arataca, Carrapicho, Catuama e Maniquara, representando a região com a maior produção pesqueira do estado, com mais de $20 \%$ do total, e cujas características são as produções artesanais de trabalho familiar e comunitário (MOURA, 2009; COUTINHO et al., 2018; LIMA; ANDRADE, 2018b).

\section{Coleta dos dados}

No total, foram realizadas 14 coletas entre os meses de julho de 2013 e junho de 2014, em um dia de cada mês, exceto em outubro de 2013 e maio de 2014, quando houve dois dias de coletas. As amostras foram obtidas por um pescador selecionado aleatoriamente, mas com experiência na utilização da rede de emalhar conhecida localmente como "redinha". O petrecho tem tamanho de malha de $30 \mathrm{~mm}$ entre nós opostos, constituído com fio de nylon monofilamento, a tralha superior com flutuadores e a inferior com chumbos, e dimensões variáveis de altura (2,2 a 2,5 m) e comprimento ( 150 a $200 \mathrm{~m}$ ). As capturas ocorreram no período matutino, horário em que a maioria das pescarias é realizada (ANDRADE; SILVA, 2013), em ciclos de marés variados e com a captura dos peixes decorrente de um lance de pesca.

As pescarias foram efetuadas aleatoriamente no CSC em profundidades que variaram entre 3 e $6 \mathrm{~m}$. Os peixes capturados foram retirados da rede um por vez, para que fossem efetuados os registros das posições (Figura 2). Na posição no sentido transversal do canal, o comprimento da rede foi dividido entre margens e centro. As extremidades da rede corresponderam às regiões de margens, que poderiam ser próximas ao município de Ilha de Itamaracá ou Itapissuma. A porção central do petrecho ou uma das extremidades ficaram dispostas no leito do canal. Na posição vertical da rede, que atua mais na camada superficial da água, foi feita a divisão em três partes iguais para registros, sendo a superior, próxima à superficie e da tralha com flutuadores, a do meio e a inferior, com proximidade da tralha com chumbos, assim correspondendo a diferentes profundidades. Os espécimes obtidos foram acondicionados em sacos plásticos, etiquetados segundo a classificação descrita

FIGURA 2: Esquema de classificação quanto ao posicionamento em que o peixe ficou retido na rede de emalhar na pesca no Canal de Santa Cruz.

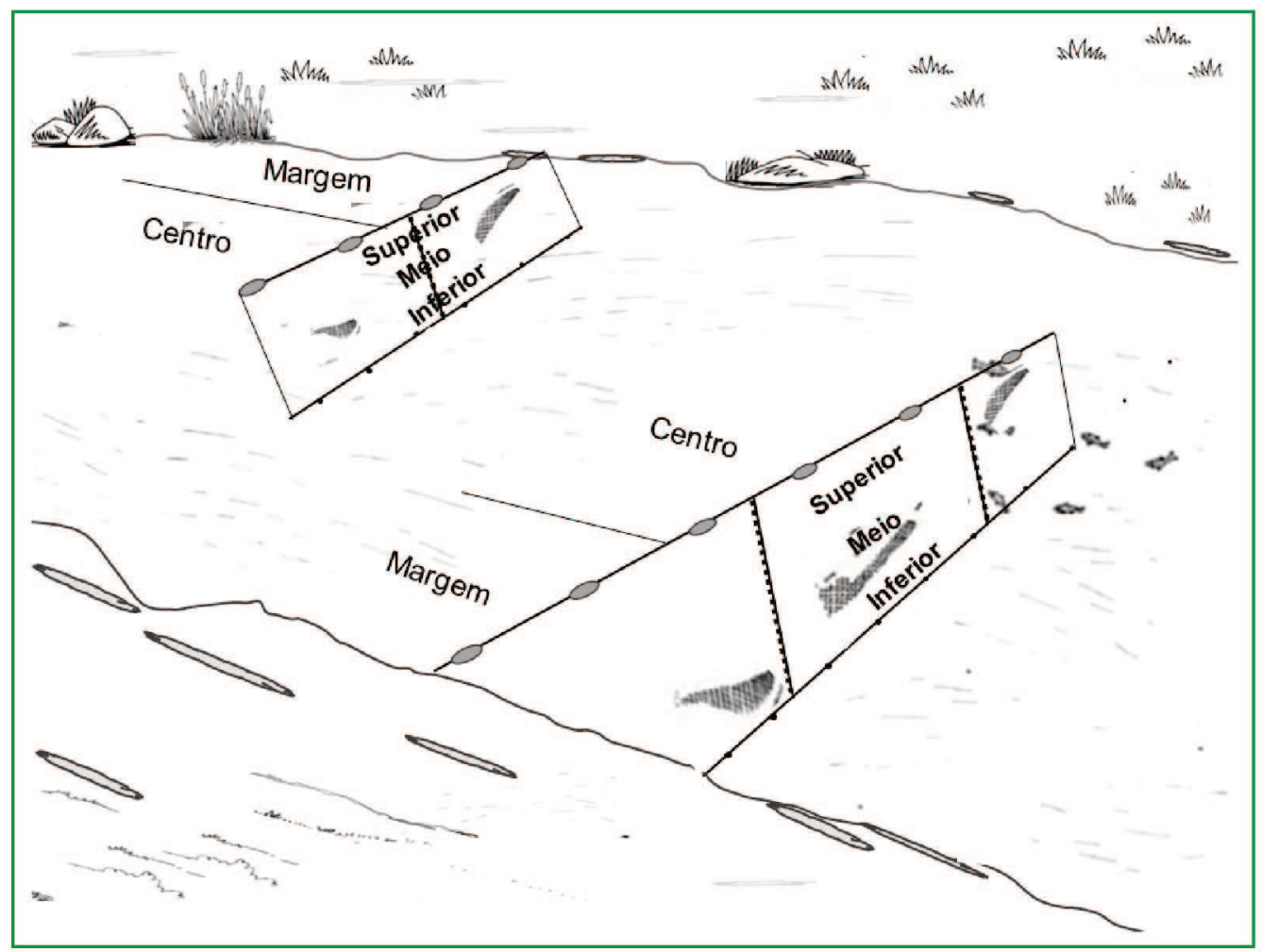


anteriormente e levados para a Universidade Federal Rural de Pernambuco (UFRPE), onde posteriormente foram identificados até o nível de espécie, com a utilização de chaves taxonômicas (FIGUEIREDO; MENEZES, 1978; CARPENTER, 2002).

\section{Análise dos dados}

Os períodos de seca e chuva no CSC foram verificados através dos dados da Agência Pernambucana de Águas e Clima (APAC), de acordo com a pluviosidade em duas estações meteorológicas da região, localizadas nos municípios de Ilha de Itamaracá e Itapissuma (MEDEIROS; KJERFVE, 1993), e assim foi possível comparar a ictiofauna nos dois momentos.

As pluviosidades mensais, geralmente, foram mais elevadas em Itapissuma (Figura 3). O mês foi considerado chuvoso quando apresentou a média da pluviosidade acima de $110 \mathrm{~mm}$, e esse valor ocorreu nos meses de julho a setembro de 2013 e de fevereiro a junho de 2014, sendo assim um período com maior amplitude de meses, quando comparado ao de seca, que ficou entre outubro de 2013 a janeiro de 2014.
A frequência de ocorrência de cada espécie foi calculada para todos os dados agregados, considerando os estratos sazonais (chuva e seca) e também os espaciais, que denotam a posição em que os exemplares foram retidos na rede e consequentemente no CSC (centro, margem, superior, meio e inferior). As espécies foram classificadas de acordo com a frequência calculada, como descrito por Dajoz (1973), em que as espécies "comuns" estavam presentes em mais de 50\% das amostras, as espécies "acessórias" entre 25 e $50 \%$ das amostras e as espécies "ocasionais" presentes em menos de $25 \%$ das amostras.

$\mathrm{Na}$ estimativa da riqueza, considerando o número de espécies nas amostras realizadas, foi empregado o método de curvas de acumulação do observado e reamostragens com Jackknife 1 e Bootstrap.

$\mathrm{Na}$ análise da diversidade alfa, foram estimados os índices de Shannon-Weaver (SHANNON; WEAVER, 1949), Simpson (SIMPSON, 1949) e Pielou (PIELOU, 1969), esses índices são geralmente aplicados com o intuito de avaliar as discrepâncias de comunidades. O índice de Shannon-Weaver é frequentemente utilizado quando a comunidade inteira não pode ser inventariada,

FIGURA 3: Pluviometria mensal dos municípios de Ilha de Itamaracá, Itapissuma e média entres os dois nos meses de julho de 2013 a junho de 2014 de acordo com os dados da Agência pernambucana de águas e clima (Apac).

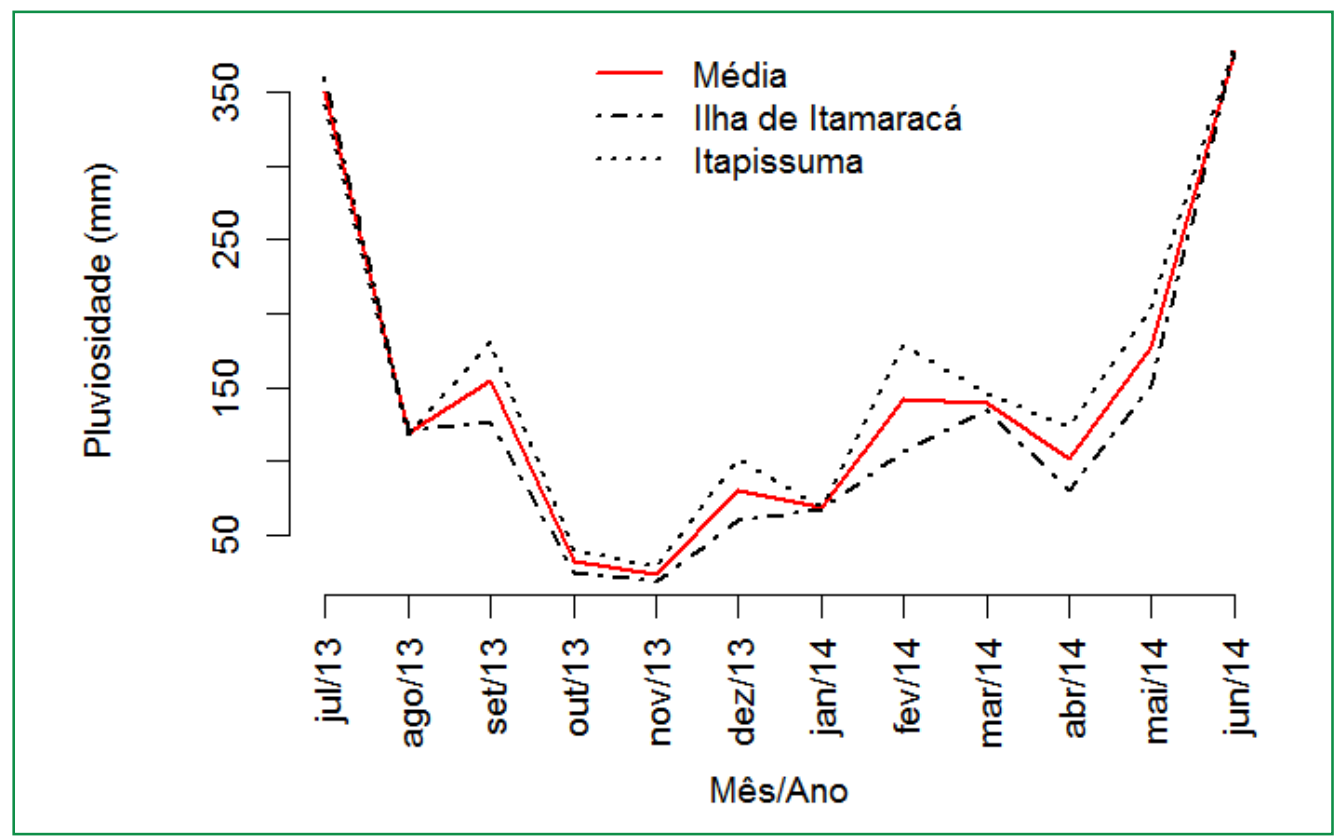


assumindo-se que as amostras foram realizadas sobre uma população "infinitamente grande". O índice de Simpson corresponde ao nível de dominância e a derivação desse cálculo está relacionada à probabilidade de dois indivíduos escolhidos ao acaso na comunidade pertencerem à mesma espécie. $\mathrm{O}$ índice de Pielou expressa a equidade entre as diferentes espécies. Valores altos de Shannon-Weaver e Pielou representam uma qualidade ecológica mais elevada, enquanto o índice de Simpson indica o oposto. As estimativas foram efetuadas considerando os estratos sazonais (chuva e seca) e os dois estratos espaço-temporais, sendo um nos trechos do $\mathrm{CSC}(\mathrm{CCH}$ - centro na chuva; $\mathrm{MCH}$ - margem na chuva; CSE - centro na seca; MSE - margem na seca) e o segundo nos setores verticais da rede, que denotam a profundidade de distribuição $(\mathrm{SCH}$ - superior na chuva; $\mathrm{MECH}$ - meio na chuva; ICH - inferior na chuva; SSE - superior na seca; MESE - meio na seca; ISE inferior na seca). Nos cálculos da diversidade beta foram considerados apenas os estratos espaço-temporais. Foi calculado o índice de similaridade binário de Jaccard, que expressa a semelhança entre ambientes, baseando-se na proporção das espécies compartilhadas entre as amostras em relação ao número total de espécies. Adicionalmente, foi realizada uma análise de agrupamento utilizando-se o método da ligação média entre grupos (UPGMA), com base na dissimilaridade de Bray-Curtis, para comparar a abundância da icitiofauna nas amostras realizadas.

Nas estimativas dos testes de hipóteses realizadas foram verificados os pressupostos de normalidade e homocedasticidade para utilização de inferências paramétricas, mas como não foram atendidos, optou-se pelo uso de testes não paramétricos $(\alpha=0,05)$ de MannWhitney, quando se comparou os estratos temporais, e Kruskal-Wallis, quando foram avaliados os estratos espaço-temporais. Em casos em que se verificaram diferenças nos testes de Kruskal-Wallis, testes da diferença mínima significativa (testes de Fisher) foram aplicados. Todas as análises foram realizadas com o programa $\mathrm{R}$, e as diversidades alfa e beta foram obtidas com auxílio do pacote vegan (OKSANEN et al., 2018; R CORE TEAM, 2020).

\section{Resultados}

Nas amostras realizadas, foram capturadas 19 espécies, pertencentes a nove famílias e, entre estas, as mais abundantes foram Carangidae, Engraulidae e Gerridae, cada uma com quatro espécies identificadas (Tabela 1). Em relação à frequência de ocorrência total no CSC, 11 espécies foram consideradas ocasionais, cinco acessórias e apenas três constantes. Nos períodos, as espécies ocasionais foram maiores na seca (15) do que na chuva (12). Nos estratos espaciais, as consideradas ocasionas foram mais presentes no trecho margem (17) do que no centro (17). Já nos setores verticais da rede o número foi crescente no setor superior (15), meio (16) e inferior (17), respectivamente. As espécies $O$. oglinum e A. clupeoides se destacaram por serem as únicas constantes em todos os estratos temporais e espaciais.

A riqueza da ictiofauna no CSC com as 14 amostras realizadas não atingiu claramente uma assíntota (Figura 4), ou seja, a riqueza total observada $(n=19)$ possivelmente seria maior com a elevação do número de amostras, o que foi também perceptível quando consideradas as estimativas com Jackkinife $1 \mathrm{e}$ Bootstrap.

As diversidades alfas estimadas nas estações não apresentaram diferenças significativas entre as estações de chuva e seca para os índices de Shannon $(U=27$, $p=0,61)$, Simpson $(U=28, p=0,52)$ e Pielou $(U=21$, $\mathrm{p}=0,90$ ) (Figuras $5 \mathrm{~A}, 5 \mathrm{~B}$ e $5 \mathrm{C}$ ), porém, foram mais variadas e com valores de medianas maiores para todos na estação de chuva.

Nos trechos analisados, considerando a divisão por estação, as estimativas não diferiram significativamente para os índices de Shannon $\left(\chi^{2}=0,09 ; \mathrm{p}=0,99\right.$; $\mathrm{gl}=3)$, Simpson $\left(\chi^{2}=0,21 ; \mathrm{p}=0,98 ; \mathrm{gl}=3\right)$ e Pielou $\left(\chi^{2}=0,03 ; \mathrm{p}=0,99 ; \mathrm{gl}=3\right)$ (Figuras 6A, 6B e 6C). De maneira geral, as medianas de todos os índices foram maiores na estação de chuva, tanto no centro como na margem. 
TABELA 1: Frequência de ocorrência das espécies capturadas com rede de emalhar no Canal de Santa Cruz, por período, trecho, setor vertical da rede e total. As letras a, o e c são para as espécies classificadas como acessórias, ocasionais e constantes, respectivamente.

\begin{tabular}{|c|c|c|c|c|c|c|c|c|c|c|}
\hline \multirow{3}{*}{ Família } & \multirow{3}{*}{ Espécie } & \multicolumn{8}{|c|}{ Frequência de Ocorrência (\%) } & \multirow{3}{*}{$\begin{array}{c}\mathrm{N} \\
(\%)\end{array}$} \\
\hline & & \multicolumn{2}{|c|}{ Período } & \multicolumn{2}{|c|}{ Trecho } & \multicolumn{3}{|c|}{ Setor } & \multirow{2}{*}{ Total } & \\
\hline & & Chuva & Seca & Centro & Margem & Superior & Meio & Inferior & & \\
\hline Belonidae & $\begin{array}{l}\text { Strongylura marina (Walbaum, } \\
1792 \text { ) }\end{array}$ & $33(a)$ & $60(\mathrm{c})$ & $15(0)$ & $15(0)$ & 43(a) & $0(0)$ & $0(0)$ & $43(\mathrm{a})$ & 0,28 \\
\hline \multirow{4}{*}{ Carangidae } & Caranx crysos (Mitchill, 1815) & $22(0)$ & $0(0)$ & $15(0)$ & $0(0)$ & $7(0)$ & $7(0)$ & $7(0)$ & $14(0)$ & 0,04 \\
\hline & $\begin{array}{l}\text { Chloroscombrus chrysurus } \\
\text { (Linnaeus, 1766) }\end{array}$ & $44(a)$ & $0(0)$ & $15(0)$ & $15(0)$ & 14(o) & $14(0)$ & $14(0)$ & 29(a) & 0,87 \\
\hline & Oligoplites palometa (Cuvier, 1832) & $11(\mathrm{o})$ & $40(a)$ & $8(0)$ & $15(\mathrm{o})$ & $7(0)$ & $14(0)$ & $7(0)$ & $21(0)$ & 0,04 \\
\hline & $\begin{array}{l}\text { Oligoplites saurus (Bloch e } \\
\text { Schneider, 1801) }\end{array}$ & 33(a) & $20(0)$ & $15(0)$ & $23(0)$ & $14(0)$ & $14(0)$ & $7(0)$ & 29(a) & 0,10 \\
\hline Centropomidae & $\begin{array}{l}\text { Centropomus undecimalis (Bloch, } \\
\text { 1792) }\end{array}$ & $22(0)$ & $0(0)$ & $8(0)$ & $0(0)$ & $0(0)$ & $0(0)$ & $7(0)$ & $14(0)$ & 0,01 \\
\hline \multirow[b]{2}{*}{ Clupeidae } & Harengula clupeola (Cuvier, 1829) & $44(a)$ & $0(0)$ & $23(0)$ & $15(0)$ & $21(0)$ & $21(0)$ & $21(0)$ & $29(\mathrm{a})$ & 0,46 \\
\hline & $\begin{array}{l}\text { Opisthonema oglinum (Le Sueur, } \\
\text { 1818) }\end{array}$ & $89(c)$ & $100(\mathrm{c})$ & $100(c)$ & $100(c)$ & $100(\mathrm{c})$ & $100(c)$ & $100(\mathrm{c})$ & $93(\mathrm{c})$ & 90,47 \\
\hline Dactylopteridae & $\begin{array}{l}\text { Dactylopterus volitans (Linnaeus, } \\
\text { 1758) }\end{array}$ & $0(0)$ & $20(0)$ & $0(0)$ & $8(0)$ & $0(0)$ & $0(\mathrm{o})$ & $7(0)$ & $7(0)$ & 0,01 \\
\hline \multirow{4}{*}{ Engraulidae } & $\begin{array}{l}\text { Anchovia clupeoides (Swainson, } \\
\text { 1839) }\end{array}$ & $89(c)$ & $100(\mathrm{c})$ & $85(c)$ & $77(\mathrm{c})$ & $79(\mathrm{c})$ & 86(c) & $85(\mathrm{c})$ & $93(c)$ & 3,50 \\
\hline & $\begin{array}{l}\text { Anchoa tricolor (Spix e Agassiz, } \\
\text { 1829) }\end{array}$ & $22(0)$ & $0(0)$ & $8(0)$ & $0(0)$ & $7(0)$ & $0(0)$ & $0(0)$ & $14(0)$ & 0,02 \\
\hline & $\begin{array}{l}\text { Cetengraulis edentulus (Cuvier, } \\
\text { 1829) }\end{array}$ & $11(0)$ & $20(0)$ & $8(0)$ & $15(0)$ & $21(0)$ & $14(0)$ & $14(0)$ & $7(0)$ & 1,18 \\
\hline & $\begin{array}{l}\text { Lycengraulis grossidens (Spix e } \\
\text { Agassiz, 1829) }\end{array}$ & $67(\mathrm{c})$ & $20(0)$ & 31(a) & $23(0)$ & $36(\mathrm{a})$ & $36(a)$ & $21(0)$ & $50(\mathrm{c})$ & 2,67 \\
\hline \multirow{4}{*}{ Gerreidae } & Diapterus auratus Ranzani, 1842 & $11(0)$ & $0(0)$ & $0(0)$ & $0(0)$ & $0(0)$ & $7(0)$ & $7(0)$ & $7(0)$ & 0,05 \\
\hline & Diapterus rhombeus (Cuvier, 1829) & $22(0)$ & $20(0)$ & $8(0)$ & $15(0)$ & $0(0)$ & $7(0)$ & $21(0)$ & $21(0)$ & 0,10 \\
\hline & $\begin{array}{l}\text { Eucinostomus argenteus } \\
\text { Baird e Girard, } 1855\end{array}$ & $22(0)$ & $40(a)$ & $8(0)$ & $23(0)$ & $7(0)$ & $7(0)$ & $21(0)$ & $29(\mathrm{a})$ & 0,15 \\
\hline & $\begin{array}{l}\text { Eucinostomus gula (Quoy e } \\
\text { Gaimard, 1824) }\end{array}$ & $22(0)$ & $0(0)$ & $8(0)$ & $0(0)$ & $0(0)$ & $0(0)$ & $14(0)$ & $14(0)$ & 0,02 \\
\hline Sciaenidae & Bairdiella ronchus (Cuvier, 1830) & $11(0)$ & $0(0)$ & $8(0)$ & $0(0)$ & $0(0)$ & $7(0)$ & $0(0)$ & $7(0)$ & 0,01 \\
\hline Scombridae & $\begin{array}{l}\text { Scomberomorus brasiliensis } \\
\text { Collette, Russo e Zavala-Camin, } \\
1978\end{array}$ & $11(0)$ & $0(0)$ & $0(\mathrm{o})$ & $0(0)$ & $0(\mathrm{o})$ & $0(\mathrm{o})$ & $0(0)$ & 7(o) & 0,02 \\
\hline
\end{tabular}


FIGURA 4: Curvas de rarefação da ictiofauna capturada no Canal de Santa Cruz com os valores observados e com estimadores Jackkinife 1 e Bootstrap.

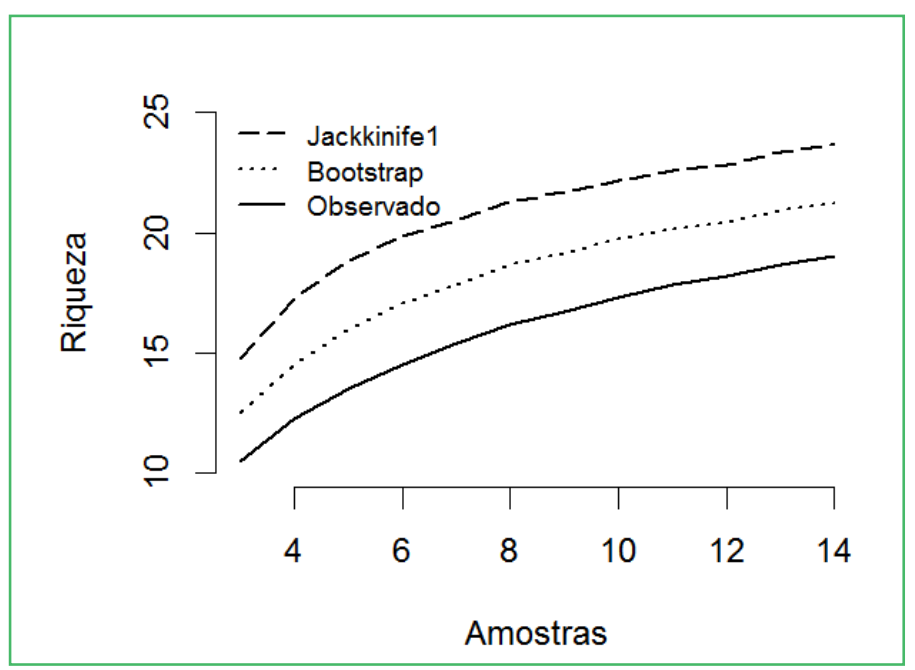

FIGURA 5: Diagrama de caixas com as estimativas das diversidades de Shannon (A), Simpson (B) e Pielou (C) da ictiofauna capturada nas estações de chuva e seca no Canal de Santa Cruz.

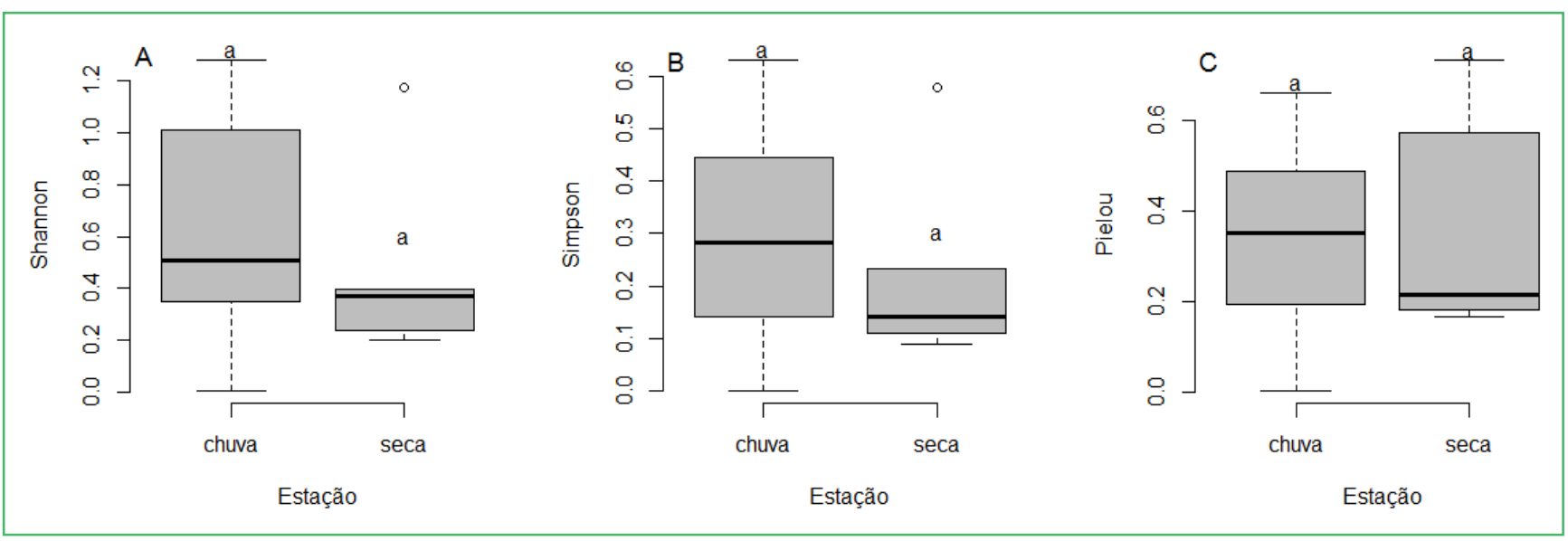

FIGURA 6: Diagrama de caixas com as estimativas das diversidades de Shannon (A), Simpson (B) e Pielou (C) da ictiofauna capturada nos trechos de centro e margem no Canal de Santa Cruz e separados por estação de chuva e seca. $\mathrm{CCH}=$ centro na chuva; $\mathrm{MCH}=$ margem na chuva; $\mathrm{CSE}=$ centro na seca; $\mathrm{MSE}=$ margem na seca.

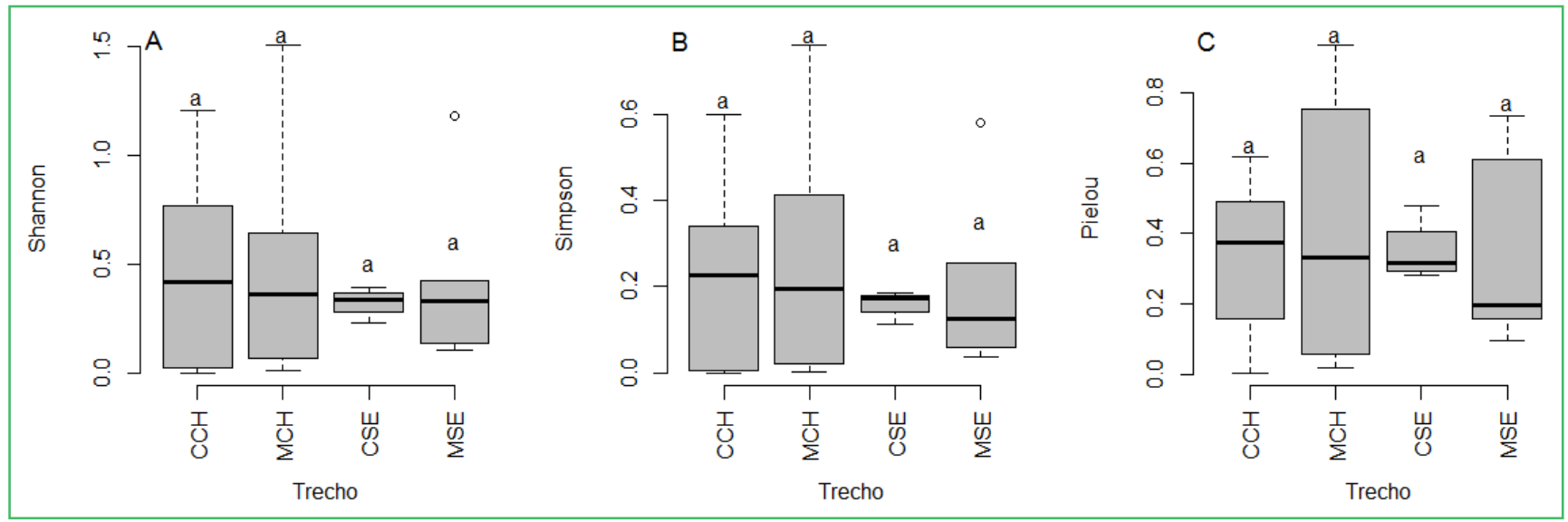


Nos setores da rede em que as espécies de peixes ficaram retidas e estes divididos por estação, não ocorreram diferenças significativas para os índices de Shannon $\left(\chi^{2}=2,34 ; \mathrm{p}=0,80 ; \mathrm{gl}=5\right)$, Simpson $\left(\chi^{2}=1,90 ; \mathrm{p}=0,86 ; \mathrm{gl}=5\right)$ e Pielou $\left(\chi^{2}=1,91 ; \mathrm{p}=0,86\right.$; $\mathrm{gl}=5$ ) (Figuras 7A, 7B e 7C). As medianas de todos os índices foram maiores nos setores do meio e inferior na estação de chuva, sendo que para o segundo os valores foram superiores, comparado a todos os outros. $\mathrm{Na}$ região superior da rede, as medianas dos índices foram mais elevadas no período de seca.

A diversidade de Jaccard estimada entre os trechos do CSC nos diferentes períodos foram maiores entre os estratos $\mathrm{MCH}$ e MSE, e $\mathrm{MCH}$ com $\mathrm{CCH}$, respectivamente, já os menores valores foram entre $\mathrm{CCH}$ e CSE, e MCH com CSE (Tabela 2).

Nas estimativas da beta diversidade de Jaccard, considerando os setores de profundidade da rede nos diferentes períodos, os maiores valores foram entre os estratos MECH e ICH, e MECH com SCH e ICH, respectivamente; já os menores valores estimados foram entre SCH e ISE, e SCH com MESE (Tabela 3).

$\mathrm{Na}$ análise de agrupamento com dissimilaridade de Bray-Curtis, ocorreram dois grupos distintos, o primeiro e menor composto pelas coletas 10 e 13, enquanto as demais coletas ficaram no segundo grupo, que foi o maior (Figura 8). O segundo grupo ainda pode ser dividido em três subgrupos, sendo o primeiro apenas com a coleta 5 , o segundo com quatro coletas $(2,1,3$ e 7) e o terceiro com sete coletas $(9,6,4,8,14,11$ e 12).

TABELA 2: Estimativas da diversidade Beta de Jaccard nos trechos do Canal de Santa Cruz separados por estação. $\mathrm{CCH}$ - centro na chuva; $\mathrm{MCH}-$ margem na chuva; CSE - centro na seca; MSE - margem na seca.

\begin{tabular}{cccc}
\hline Estratos & \multicolumn{3}{c}{$\begin{array}{c}\text { Diversidade de Jaccard } \\
\text { (Trecho) } \\
\text { MCH }\end{array}$} \\
& CCH & CSE \\
\hline MCH & 0,562 & & \\
CSE & 0,250 & 0,364 & \\
MSE & 0,471 & 0,667 & 0,500 \\
\hline
\end{tabular}

TABELA 3: Estimativas da diversidade beta de Jaccard nos setores verticais da rede nas capturas realizadas no Canal de Santa Cruz e separados por estação. $\mathrm{SCH}$ - superior na chuva; $\mathrm{MECH}$ - meio na chuva; ICH - inferior na chuva; SSE - superior na seca; MESE - meio na seca; ISE - inferior na seca.

\begin{tabular}{cccccc}
\multirow{2}{*}{ Estratos } & \multicolumn{5}{c}{ Diversidade de Jaccard (Setor) } \\
\cline { 2 - 6 } & SCH & MECH & ICH & SSE & MESE \\
\hline MECH & 0,533 & & & & \\
ICH & 0,533 & 0,733 & & & \\
SSE & 0,500 & 0,500 & 0,400 & & \\
MESE & 0,182 & 0,231 & 0,143 & 0,375 & \\
ISE & 0,141 & 0,357 & 0,267 & 0,400 & 0,500 \\
\hline
\end{tabular}

FIGURA 7: Diagrama de caixas com as estimativas das diversidades de Shannon (A), Simpson (B) e Pielou (C) da ictiofauna capturada nos setores superior, meio e inferior da rede de emalhar utilizada nas capturas no Canal de Santa Cruz e separados por estação de chuva e seca. $\mathrm{SCH}=$ superior na chuva; $\mathrm{MECH}=$ meio na chuva; $\mathrm{ICH}=$ inferior na chuva; $\mathrm{SSE}=$ superior na seca; MESE $=$ meio na seca; ISE $=$ inferior na seca.

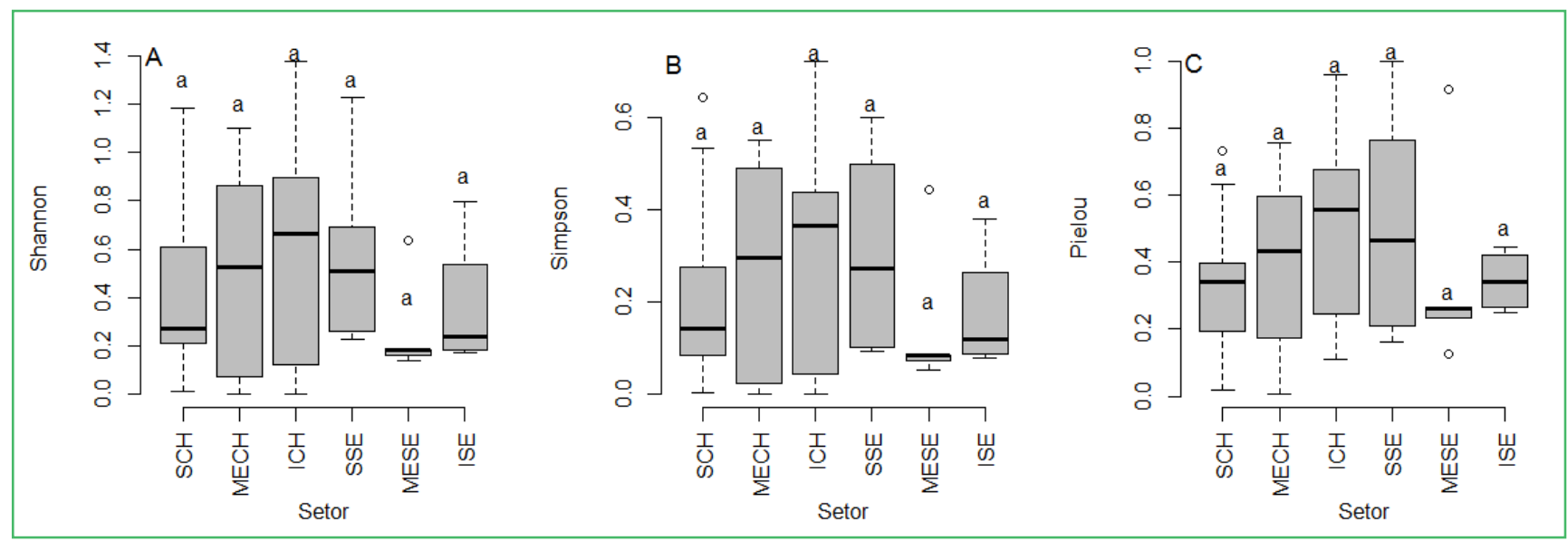


FIGURA 8: Análise de agrupamento com dissimilaridade de Bray-Curtis nas amostras realizadas com rede de emalhar no Canal de Santa Cruz.

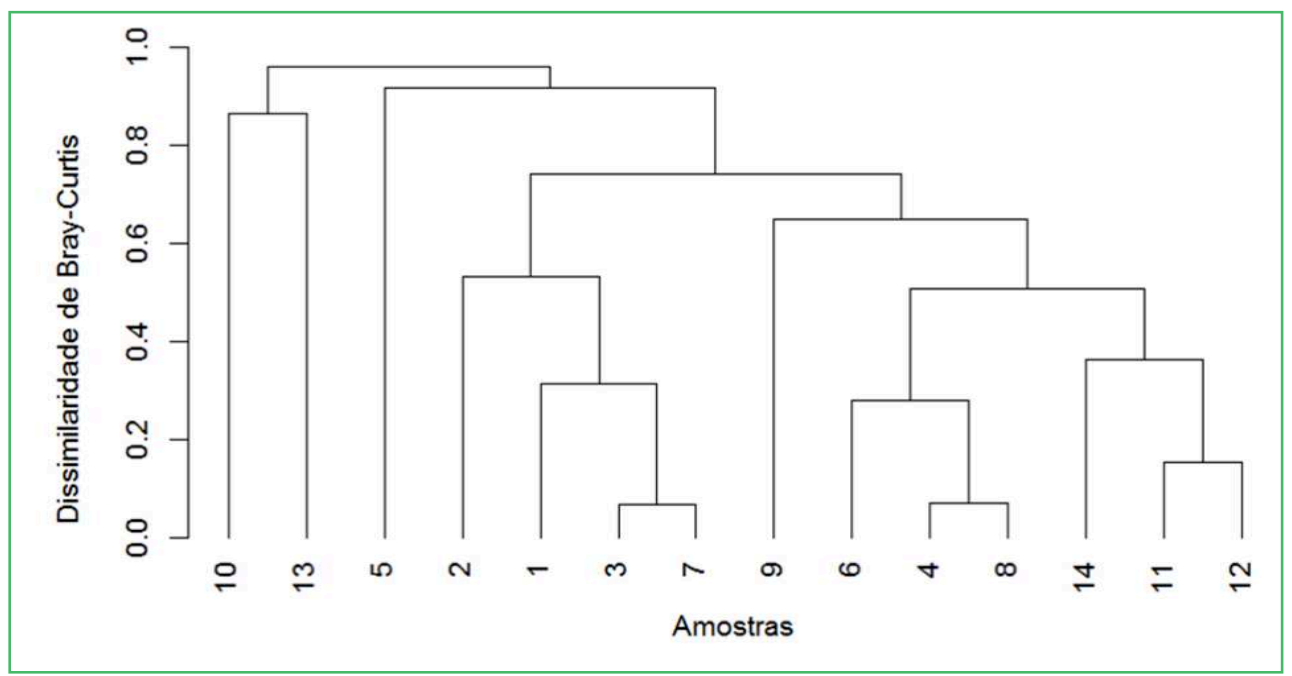

\section{Discussão}

A riqueza encontrada neste trabalho foi baixa e não atingiu uma assíntota. É evidente que a quantidade de espécies de peixes no CSC é superior à estimada neste trabalho, sendo constatado em outros levantamentos mais antigos ou mesmo com dados mais recentes, que obtiveram 145 e 141 espécies, respetivamente (VASCONCELOS FILHO; OLIVEIRA, 1999; FERREIRA et al., 2019). No entanto, ressalta-se que as espécies de peixes encontradas nas amostras foram classificadas pela International Union for the Conservation of Nature (IUCN Red List) com status de seguras ou pouco preocupantes (FROESE; PAULY, 2019), ou seja, mesmo as espécies com importância comercial estão com estoques relativamente em bom estado, de maneira global.

Ariqueza da ictiofauna e as abundâncias das espécies variam de acordo com a estação, a localização em que a amostragem é efetuada no estuário e a seletividade das artes de pesca utilizadas (VILLARROEL, 1994). Embora todos os meses tenham sido contemplados, assim como a aleatoriedade espacial, e o petrecho utilizado apresentar uma seletividade considerada ampla para várias espécies (e.g. A. clupeoides, O. oglinum), este, porém, é mais eficiente para um menor número de espécies se comparado a outras redes de emalhar com tamanho de malha superior (LIMA; ANDRADE, 2018a). No entanto, as capturas realizadas com a rede de emalhar utilizada foram suficientes para detectar que as famílias mais abundantes e comumente encontradas no CSC seguiram o mesmo padrão que em outros estuários tropicais e subtropicais com grande presença das famílias Carangidae, Engraulidae e Gerridae (ANDRADETUBINO et al., 2008; BOT NETO et al., 2018). Esse padrão corroborou o estudo apresentado por Ferreira et al. (2019) no CSC e em uma região estuarina no extremo sul de Pernambuco (FAVERO et al., 2019), mas em ambos apenas a Carangidae não ficou entre as principais.

As espécies de peixes marinhas quanto aos padrões migratórios e de exploração do ambiente estuarino, ao longo do ciclo de vida ou parte dele, podem ser consideradas residentes, visitantes ou dependentes (ELLIOTT et al., 2007). Entre as espécies identificadas no presente trabalho, seis são marinhas visitantes $(C$. chrysurus, H. clupeola, O. oglinum, D. volitans, $C$. edentulus e $S$. brasiliensis) e as 13 demais são marinhas dependentes (VASCONCELHOS FILHO; OLIVEIRA, 1999; FROESE; PAULY, 2019). Aparentemente, não ocorreu uma correlação entre a frequência de ocorrência e a relação da espécie com o estuário, pois espécies dependentes não foram mais frequentes que as visitantes. Essa falta de correlação pode estar ligada a vários fatores, inclusive os reprodutivos, pois as espécies $O$. oglinum e 
A. clupeoides, que foram constantes em todos os estratos avaliados, são marinhas visitantes e dependentes, respectivamente, mas as desovas ocorrem durante praticamente todo o ano (CASELLES; ACERO, 1996; PEÑA-ALVARO et al., 2009). Assim há uma constância no recrutamento de indivíduos jovens durante todo o ano no CSC (LIMA; ANDRADE, 2018a), o que permite que essas espécies sejam frequentes. O caso oposto, com baixa frequência, ocorreu para C. undecimalis (marinha dependente) e $D$. volitans (marinha visitante), cujas atividades reprodutivas acontecem durante poucos meses do ano (MACHADO et al., 2002; PERERA-GARCÍA et al., 2011).

As frequências de ocorrência nos estratos temporais e espaciais não apresentaram diferenças preponderantes, principalmente para os espaciais. No período chuvoso ocorreu um maior número de espécies não classificadas como ocasionais, quando comparado ao período de seca, e essa tendência apresentada poderia ser mais acentuada devido ao aporte de nutrientes que ocorre com o aumento da vazão em regiões estuarinas nessa época, que também oferece um maior espaçamento para abrigos contra predadores (BLABER, 2000), mas a diferença não foi evidente possivelmente pelo número baixo de coletas. Nos estratos espaciais, as frequências foram de menores possibilidades de espécies ocasionais no centro do CSC e na região superior da rede.

A grande proporção de $O$. oglinum foi em decorrência de alguns fatores, o primeiro e já anteriormente mencionado foi em relação ao petrecho (seletividade e eficiência), o segundo diz respeito ao conhecimento empírico dos pescadores em relação à dinâmica no CSC, que conseguem detectar os cardumes de O. oglinum, espécie alvo das capturas na localidade. Além disso, as assembleias de peixes em ambientes estuarinos são dominadas por espécies que incluem as famílias Clupeidae e Engraulidae (MONTELEONE, 1992; RAMOS et al., 2006; KINDONG et al., 2020). Na família Engraulide, a espécie A. clupeoides foi a que mais se destacou e larvas dessa família foram dominantes no CSC (EKAU et al., 2001), o que justifica o aparecimento de algumas espécies entre as mais capturadas, apesar de elas não serem alvo, o que muitas vezes acarreta capturas subaproveitadas ou mesmo descartes no porto por não terem valor comercial (LIMA, observação pessoal).

A clara dominância da espécie $O$. oglinum afetou as estimativas dos índices de diversidade, tanto nos estratos temporais quanto nos espaciais. $\mathrm{Na}$ diversidade de Shannon-Weaver, geralmente, os valores calculados são subestimados em situações em que as comunidades de peixes têm espécies dominantes (SALAS et al. 2006; KAMRANI et al., 2015; KINDONG et al., 2020). Os valores dos índices de Simpson e Pielou foram baixos neste trabalho, principalmente nas ocasiões em que $O$. oglinum predominou, assim evidenciando a dominância desta e a baixa equidade da distribuição entre as espécies, respectivamente.

Nos períodos de chuva e seca os índices não apresentaram diferenças significativas, ocorrendo padrão similar entre marés de cheia e de vazante em um estuário em Sergipe (Nordeste do Brasil) (ARAÚJO et al., 2017). Em relação à variação mais acentuada da diversidade na estação chuvosa, esta pode ser reflexo de um aumento da frequência das correntes de retorno, enquanto no período de seca o estuário tem características mais homogêneas, resultantes de águas marinhas entrando na maior parte do ambiente (PAIVA et al., 2009). Valores maiores de diversidade com diferenças significativas também são encontrados na estação chuvosa, como, por exemplo, no sistema estuarino de Santos, em São Paulo (Sudeste do Brasil) (SANTOS et al., 2015). O regimento das diversidades nos estratos espaciais foi levemente influenciado pelo fator sazonal. No centro e na margem do CSC ocorreram maiores diversidades e equidade das espécies no período chuvoso, e menor dominância de O. oglinum. Considerando a divisão vertical da rede, os setores do meio e inferior apresentaram o mesmo padrão, enquanto no superior a maior ocorreu na seca.

A diversidade beta com valor maior na margem do CSC em diferentes períodos (MCH e MSE) ocorreu pela similaridade da composição da ictiofauna nesses estratos, pois, em ambos, um total de 10 espécies foram capturadas, sendo oito comuns entre os dois e o número de indivíduos de O. oglinum variou entre 1300 e 1800. Nos estratos com maiores distinções, que foram no centro do canal na chuva e seca (CCH e $\mathrm{CSE}$ ), o número total de espécies foi de 15 em $\mathrm{CCH}$ e cinco em CSE, 
sendo quatro comuns em ambos, e, adicionalmente, no primeiro ocorreu uma quantidade de espécimes capturados de $O$. oglinum (4735) muito superior ao segundo (859). Nos estratos espaciais em que foram considerados os setores verticais da rede, os valores da diversidade beta demonstraram maiores igualdades entre as comunidades de peixes encontradas na parte do meio e inferior da rede na chuva (MECH e ICH), com 13 espécies capturadas; dessas, 11 foram comuns nos dois estratos, e as capturas de O. oglimum variaram de 3472 (MECH) e 1332 (ICH), enquanto nos estratos com menores similaridades (SCH e ISE) os totais de espécies identificas foram de 10 ( $\mathrm{SCH}$ ) e seis (ISE), com duas comuns em ambas, e as capturas de $O$. oglinum foram de 2336 e 713 indivíduos, respectivamente.

No agrupamento entre as coletas, dois grandes grupos se distinguiram, principalmente por causa do número de exemplares capturados das espécies $O$. oglinum e L. grossidens. No menor grupo, em duas coletas (10 e 13) ocorreu captura baixa ou mesmo ausência de O. oglinum e altas capturas de L. grossidens, enquanto no maior grupo, com as demais coletas, o padrão foi o oposto, com exceção da coleta 5, que teve baixa captura de ambas as espécies. Na divisão do segundo grupo, a coleta 5 ficou isolada pelas características mencionadas anteriormente, no segundo subgrupo (2, 1, 3 e 7) houve coletas com grande presença de $O$. oglinum, variando entre 959 e 3946 indivíduos, e no terceiro subgrupo ( 9 , $6,4,8,14,11$ e 12) ocorreram capturas dessa espécie entre 45 e 524 indivíduos.

A composição sazonal e espacial da ictiofauna nas capturas efetuadas com a rede de emalhar avaliada não apresentou diferenças significativas. A redinha é um petrecho eficiente para a espécie alvo $O$. oglinum e cujas capturas de outras espécies corresponderam a menos de $13 \%$ da icitiofauna já encontrada no CSC, e estas foram pouco abundantes. Portanto, medidas de gestão que visem restringir o uso da redinha para a proteção da diversidade, considerando a sazonalidade e espacialidade no canal, não são recomendadas, porém os impactos ecológicos do uso desse tipo de rede podem ocorrer em decorrência do aumento do esforço, já que não há legislação vigente para essa pescaria, que é desenvolvida com regime de livre acesso.

\section{Agradecimentos}

À Coordenação de Aperfeiçoamento de Pessoal de Nível Superior (CAPES), por conceder uma bolsa de estudo de pós-graduação no momento da pesquisa ao primeiro autor.

\section{Referências}

ALCÂNTARA, E. H. Mudanças climáticas, incertezas hidrológicas e vazão fluvial: o caso do estuário do Rio Anil. Caminhos de Geografia, Uberlândia, v. 8, n. 12, p. 158-173, 2004.

ANDRADE, H. A.; SILVA, R. M. M. Dinâmica das frotas de pesca de emalhe e linha de mão de Itapissuma - PE no Canal de Santa Cruz. Boletim Técnico Científico - CEPENE, Tamandaré, v. 19, n. 1, p. 83-91, 2013.

ANDRADE-TUBINO, M. F.; RIBEIRO, A. N. R.; VIANA, M. Organização espaço temporal das ictiocenoses demersais nos ecossistemas estuarinos brasileiros: uma síntese. Oecologia Brasiliensis, Rio de Janeiro, v. 12, n. 4, p. 640-666, 2008.

ARAÚJO, A. R. R.; SOUZA, J. M.; LIMA, R. C. D.; ABREU, E. F. S; VIRGENS, F. F.; BARBOSA, J. M. Diversidade da fauna aquática do estuário do rio Japaratuba, estado de Sergipe, Brasil. Acta of Fisheries and Aquatic Research, São Cristóvão, v. 5, n. 1, p. 33-42, 2017.

BARLETTA, M.; BARLETTA-BERGAN, A.; SAINT-PAUL, U.; HUBOLD, G. Seasonal changes in density, biomass, and diversity of estuarine fishes in tidal mangrove creeks of the lower Caete Estuary (northern Brazilian coast, east Amazon). Marine Ecology Progress Series, Luhe, v. 256, p. 217-228, 2003.

BARRETO, C. F.; SANTANA-BARRETO, M. S. Deslocamento da sardinha-bandeira (Opisthonema oglinum, Lê Sueur, 1818) no Canal de Santa Cruz, Itamaracá - Pernambuco. Anais da Universidade Federal Rural de Pernambuco, Recife, v. 5 p. 53-60, 1980.

BLABER, S. J. M. Tropical estuarine fishes: ecology, exploitation and conservation. Oxford: Blackwell Science, 2000. 372 p.

BOT NETO, R. L.; PASSOS, A. C.; SCHWARZ JUNIOR, R.; SPACH, H. L. Use of shallow areas by ichthyofauna (Teleostei) on the north-south axis of the Paranaguá Estuarine Complex, State of Paraná, Brazil. Pan-American Journal of Aquatic Sciences, Rio Grande, v. 13, n. 1, p. 64-78, 2018.

CARPENTER, K. E. FAO species identification guide for fishery purposes and American Society of Ichthyologists and Herpetologists Special Publication. In: CARPENTER, K. E. (Ed.). The living marine resources of the Western Central Atlantic: bony fishes (Acipenseridae to Grammatidae). Rome: FAO, 2002. p. 601-1374.

CASElleS, O, A.; ACERO P, A. Reproducción de Anchovia clupeiodes y Anchovia parva (Pisces: Engraulidae) en dos ciénagas del Caribe colombiano. Revista de Biología Tropical, San José, v. 44, n. 2, p. 781-793, 1996.

COUTinho, S. M. V.; SUlaiman, S. N.; CARBOne, A. S. Área de Proteção Ambiental (APA) de Santa Cruz: (re)conhecendo o valor ecológico, histórico-cultural e econômico. Recife: CPRH, 2018. 48 p. 
DAJOZ, R. Ecologia Geral. São Paulo: Vozes, 1973. 472 p.

EKAU, W.; WESTHAUS-EKAU, P.; MACÊDO, S. J.; DORRIEN, V. The larval fish fauna of the "Canal de Santa Cruz" estuary in Northeast Brazil. Tropical Oceanography, Recife, v. 29, n. 2, p. 117-128, 2001.

ELLIOTT, M.; WHITFIELD, A. K.; POTTER, C.; BLABER, S. J. M.; CYRUS, D. P.; NORDLIE, F. G.; HARRISON, T. D. The guild approach to categorizing estuarine fish assemblages: A global review. Fish and Fisheries, Vancouver, v. 8, n. 3, p. 241-268, 2007.

FAVERO, F. L. T.; ARAUJO, I. M. S.; SEVERI, W. Structure of the fish assemblage and functional guilds in the estuary of Maracaípe, Northeast coast of Brazil. Boletim do Instituto de Pesca, São Paulo, v. 45, n. 1, e.417, 2019.

FERREIRA V.; LE LOC'H F.; MÉNARD F.; FRÉDOU T.; FRÉDOU F. L. Composition of the fish fauna in a tropical estuary: the ecological guild approach. Scientia Marina, Barcelona, v. 83, n. 2, p. 1-11, 2019.

FROESE, R.; PAULY, D. FishBase. 2019. Disponível em: <https:// www.fishbase.org $>$.

HAEDRICH, R. L. Estuarine fishes. In: KETCHUM, B. H. (Ed.) Ecosystems of the world. Estuaries and enclosed seas. Amsterdam: Elsevier, 1983. p. 183-207.

HOVGÅRD, H.; LASSEN, H.; MADSEN, N.; POULSEN, T. M.; WILEMAN, D. Gillnet selectivity for North Sea Atlantic cod (Gadus morhua): Model ambiguity and data quality are related. Canadian Journal of Fisheries and Aquatic Sciences, Ottawa, v. 56, n. 8, p. 1307-1316, 1999.

JESUS, H. C.; COSTA, E. A.; MENDONÇA, A. S. F.; ZANDONADE, E. Distribuição de metais pesados em sedimentos do sistema estuarino da Ilha de Vitória-ES. Química Nova, São Paulo, v. 27, n. 3, p. 378-386, 2004.

KAMRANI, E.; SHARIFINIA, M.; HASHEMI, S. H. Analyses of fish community structure changes in three subtropical estuaries from the Iranian coastal waters. Marine Biodiversity, Heidelberg, v. 46, p. 561-577, 2015.

KINDONG, R.; WU, J.; GAO, C.; DAI, L.; TIAN, S.; DAI, X.; CHEN, J. Seasonal changes in fish diversity, density, biomass, and assemblage alongside environmental variables in the Yangtze River Estuary. Environmental Science and Pollution Research, Talance, v. 27, p. 25461-25474, 2020.

LESSA, R.; DUARTE-NETO, P.; MORIZE, E.; MACIEL, R. Otolith microstructure analysis with OTC validation confirms age overestimation in Atlantic thread herring Opisthonema oglinum from northeastern Brazil. Journal of Fish Biology, Malden, v. 73, n. 7, p. 1690-1700, 2008.

LIMA, S. A. O.; ANDRADE, H. A. Gillnet selectivity for forage fish with emphasis on manjuba (Opisthonema oglinum) in an estuary in the Northeast of Brazil. Boletim do Instituto de Pesca, São Paulo, v. 44, n. 3, p. e225, 2018 a.

LIMA, S. A. O.; ANDRADE, L. H. A. Análise da evolução do setor pesqueiro de Pernambuco. Arquivos de Ciências do Mar, Fortaleza, v. 51, n. 2, p. 27-43, 2018 b.

MACHADO, J. F.; ZAMBELI, R. M.; VIANNA, M. Biologia reprodutiva do falso-voador, Dactylopterus volitans (TELEOSTEI:
DACTYLOPTERIDAE), no litoral norte do estado de São Paulo, Brasil. Arquivos de Ciências do Mar, Fortaleza, v. 35, n. 1-2, p. 125-129, 2002.

MCERLEAN A. J.; O'CONNOR, S. G.; MIHURSKY, J. A.; GIBSON, C. I. Abundance, diversity and seasonal patterns of estuarine fish populations. Estuarine, Coastal and Shelf Science, New York, v. 1, p. 19-36, 1973.

MEDEIROS, C.; KJERFVE, B. Hydrology of a tropical estuarine system: Itamaracá, Brazil. Estuarine, Coastal and Shelf Science, New York, v. 36, n. 5, p. 495-515, 1993.

MÉRIGOT, B.; FRÉDOU, F. L.; VIANA, A. P.; FERREIRA, B. P.; JUNIOR, E. D. N. C.; SILVA JÚNIOR, C. B.; FRÉDOU, T. Fish assemblages in tropical estuaries of Northeast Brazil: A multicomponent diversity approach. Ocean \& Coastal Management, Augustinusga, v. 30, p. e9-e183, 2016.

MONTELEONE, D. M. Seasonality and abundance of ichthyoplankton in great south bay, New York. Estuaries, Chesapeake, v. 15, p. 230-238, 1992.

MONTES, M. J. F., MACÊDO, S. J.; KOENING, M. L. N:Si:P atomic ratio in the Santa Cruz Channel, Itamaracá-PE (Northeast Brazil): a nyctemeral variation. Brazilian Archives of Biology and Technology, Curitiba, v. 45, n. 2, p. 115-124, 2002.

MORENO-VALCÁRCEL， R. M.; OLIVA-PATERNA， F. J.; ARRIBAS, C.; FERNÁNDEZ-DELGADO, C. Fish composition and assemblage in the anthropogenic-modified tidally-restricted Doñana (Spain) marshlands. Estuarine Coastal and Shelf Science, London, v. 119, n. 1, p. 54-63, 2013.

MOURA, R, T. Aspectos gerais da hidrobiologia do litoral Norte de Pernambuco - Brasil. Brasília: Ibama, 2009, 138 p.

NÓBREGA, M. F.; LESSA, R.; SANTANA, F. M. Peixes marinhos da região Nordeste do Brasil. (Programa Revizee Score Nordeste). Fortaleza: Editora Martins e Cordeiro, 2009. $208 \mathrm{p}$.

OKSANEN, J.; BLANCHET, F. G.; FRIENDLY, M.; KINDT, R.; LEGENDRE, P.; MCGLINN, D.; MINCHIN, P. R.; O'HARA, R. B.; SIMPSON, G. L.; SOLYMOS, P.; STEVENS, M. H. H.; SZOECS, E.; WAGNER, H. Vegan: Community Ecology Package. 2018. Disponível em $<$ https:// CRAN.R-project.org/package=vegan/>.

PAIVA, A. C. G.; LIMA, M. F. V.; SOUZA, J. R. B.; ARAÚJO, M. E. Spatial distribution of the estuarine ichthyofauna of the Rio Formoso (Pernambuco, Brazil), with emphasis on reef fish. Zoologia, Curitiba, v. 26, n. 2, p. 266-278, 2009.

PERERA-GARCÍA, M. A.; MENDONZA-CARRANZA, M.; CONTRERAS-SÁNCHEZ, W. M.; HUERTA-ORTÍZ, M.; PÉREZ-SÁNCHEZ, E. Reproductive biology of common snook Centropomus undecimalis (Perciformes: Centropomidae) in two tropical habitats. Revista de Biología Tropical, San José, v. 59, n. 2, p. 669-681, 2011.

PEÑA-ALVARO, N.; FIGUEROLA-FERNÁNDEZ, M.; TORRES-RUIZ, W. Reproductive biology of three important baitfishes (Clupeidae) in Puerto Rico. In: PROCEEDINGS OF THE GULF AND CARIBBEAN FISHERIES INSTITUTE, 61, 2009, Guadeloupe. Proceedings... Guadeloupe: Gulf and Caribbean Fisheries Institute. 2009. p. 38-47.

PIELOU, E. C. An introduction to Mathematical Ecology. New York: Wiley Interscience, 1969. 286 p. 
POTTER, I. C.; CHUWEN, B. M.; HESP, S. A.; HALL, N. G.; HOEKSEMA, S. D.; FAIRCLOUGH, D. V.; RODWELL, T. M. Implications of the divergent use of a suite of estuaries by two exploited marine fish species. Journal of Fish Biology, Malden, v. 79, n. 3, p. 662-691, 2011.

R CORE TEAM. R: a language and environment for statistical computing. 2020. Disponível em: <https://www.R-project.org/>.

RAMOS, S.; COWEN, R. K.; RE, P.; BORDALO, A. A. Temporal and spatial distributions of larval fish assemblages in the Lima Estuary (Portugal). Estuarine, Coastal and Shelf Science, New York, v. 66, n. 1-2, p. 303-314, 2006.

RAY, G. C. Connectivities of estuarine fishes to the coastal realm. Estuarine, Coastal and Shelf Science, New York, v. 64, n. 1, p. 18-32, 2005.

SALAS, F.; MARCOS, C.; NETO, J.; PATRÍCIO, J.; PÉREZRUZAFA, A.; MARQUES, J. User-friendly guide for using benthic ecological indicators in coastal and marine quality assessment. Ocean \& Coastal Management, Augustinusga, v. 49, n. 5-6. p. 308-331, 2006.

SANTOS, J. A. P.; SCHMIEGELOW, J. M. M.; ROTUNDO, M. M.; BARRELLA, W. Composição e variação temporal da assembleia de peixes do alto sistema estuarino de Santos, São Paulo, Brasil. Boletim do Instituto de Pesca, São Paulo, v. 41, n. 4, p. 945-959, 2015.
SHANNON, C. E.; WEAVER, W. The Mathematical Theory of Communication. Urbana: University of Illinois Press, 1949. 117 p. SIMPSON, E. H. Measurement of diversity. Nature, London, v. 163 , n. 4148, p. 688, 1949.

VASCONCELOS FILHO, A. L.; OLIVEIRA, A. M. E. Composição e ecologia da ictiofauna do Canal de Santa Cruz (Itamaracá - PE, Brasil). Trabalhos Oceanográficos de Universidade Federal de Pernambuco, Recife, v. 27, n. 1, p. 101-113, 1999.

VILLARROEL, P. R. Estrutura de las comunidades de peces de la Laguna de Raya, isla de Margrita, Venezuela. Ciencias Marinas, Ensenada, v. 20, n. 1, p. 1-16, 1994.

VYAS, V.; DAMDE, V.; PARASHAR, V. Fish biodiversity of Betwa River in Madhya Pradesh, India with special reference to a sacred Ghat. International Journal of Biodiversity and Conservation, Tirana, v. 4, n. 2, p. 71-77, 2012.

WHITFIELD, A. K.; ELLIOTT, M. Fishes as indicators of environmental and ecological changes within estuaries: a review of progress and some suggestions for the future. Journal of Fish Biology, Malden, v. 61, n. 1, p. 229-250, 2002.

WHITTAKER, R. H. Evolution and measurement of species diversity. Taxon, Vienna, v. 21, n. 2-3, p. 213-251, 1972. 